\title{
ON THE SOLVABILITY OF INITIAL-VALUE PROBLEMS FOR NONLINEAR IMPLICIT DIFFERENCE EQUATIONS
}

\author{
PHAM KY ANH AND HA THI NGOC YEN
}

Received 18 February 2004

Our aim is twofold. First, we propose a natural definition of index for linear nonautonomous implicit difference equations, which is similar to that of linear differentialalgebraic equations. Then we extend this index notion to a class of nonlinear implicit difference equations and prove some existence theorems for their initial-value problems.

\section{Introduction}

Implicit difference equations (IDEs) arise in various applications, such as the Leontief dynamic model of a multisector economy, the Leslie population growth model, and so forth. On the other hand, IDEs may be regarded as discrete analogues of differentialalgebraic equations (DAEs) which have already attracted much attention of researchers.

Recently [1,3], a notion of index 1 linear implicit difference equations (LIDEs) has been introduced and the solvability of initial-value problems (IVPs), as well as multipoint boundary-value problems (MBVPs) for index 1 LIDEs, has been studied. In this paper, we propose a natural definition of index for LIDEs so that it can be extended to a class of nonlinear IDEs. The paper is organized as follows. Section 2 is concerned with index 1 LIDEs and their reduction to ordinary difference equations. In Section 3, we study the index concept and the solvability of IVPs for nonlinear IDEs. The result of this paper can be considered as a discrete version of the corresponding result of [4].

\section{Index 1 linear implicit difference equations}

Let $Q$ be an arbitrary projection onto a given subspace $N$ of dimension $m-r(1 \leqslant r \leqslant$ $m-1)$ in $\mathbb{R}^{m}$. Further, let $\left\{v_{i}\right\}_{1}^{r}$ and $\left\{v_{j}\right\}_{r+1}^{m}$ be any bases of $\operatorname{Ker} Q$ and $N$, respectively. Denote by $V=\left(v_{1}, \ldots, v_{m}\right)$ a column matrix and denote $\tilde{Q}=\operatorname{diag}\left(O_{r}, I_{m-r}\right)$, where $O_{r}$ and $I_{m-r}$ stand for $r \times r$ zero matrix and $(m-r) \times(m-r)$ identity matrix, respectively. Then $V$ is nonsingular, $Q=V \tilde{Q} V^{-1}$, and this decomposition depends on the choice of the bases $\left\{v_{i}\right\}_{1}^{m}$, that is, on $V$.

Now, suppose $N_{\alpha}$ and $N_{\beta}$ are two subspaces of the same dimension $m-r(1 \leqslant r \leqslant$ $m-1)$ in $\mathbb{R}^{m}$. Then any projections $Q_{\alpha}$ and $Q_{\beta}$ onto $N_{\alpha}$ and $N_{\beta}$ can be decomposed 
as $Q_{\alpha}=V_{\alpha} \tilde{Q} V_{\alpha}^{-1}$ and $Q_{\beta}=V_{\beta} \tilde{Q} V_{\beta}^{-1}$, respectively. Define an operator connecting two subspaces $N_{\alpha}$ and $N_{\beta}$ (connecting operator, for short) $Q_{\alpha \beta}:=V_{\alpha} \tilde{Q} V_{\beta}^{-1}$. Clearly,

$$
\begin{gathered}
Q_{\alpha \beta}=Q_{\alpha} Q_{\alpha \beta}=Q_{\alpha \beta} Q_{\beta}=Q_{\alpha} V_{\alpha} V_{\beta}^{-1}=V_{\alpha} V_{\beta}^{-1} Q_{\beta}, \\
Q_{\alpha \beta} Q_{\beta \alpha}=Q_{\alpha}, \quad Q_{\beta \alpha} Q_{\alpha \beta}=Q_{\beta} .
\end{gathered}
$$

We consider a system of LIDEs

$$
A_{n} x_{n+1}+B_{n} x_{n}=q_{n} \quad(n \geqslant 0)
$$

where $A_{n}, B_{n} \in \mathbb{R}^{m \times m}, q_{n} \in \mathbb{R}^{m}$ are given and $\operatorname{rank} A_{n} \equiv r(1 \leqslant r \leqslant m-1)$ for all $n \geqslant 0$. Let $Q_{n}$ be any projection onto $\operatorname{Ker} A_{n}, P_{n}=I-Q_{n}$ and consider decompositions $Q_{n}=$ $V_{n} \tilde{Q} V_{n}^{-1}(n \geqslant 0)$. For definiteness, we put $A_{-1}:=A_{0}, Q_{-1}:=Q_{0}, P_{-1}:=P_{0}$, and $V_{-1}:=$ $V_{0}$. Thus, the connecting operators $Q_{n-1, n}:=V_{n-1} \tilde{Q} V_{n}^{-1}$ are determined for all $n \geqslant 0$.

Recall that a linear DAE $A(t) x^{\prime}+B(t) x=q(t), t \in J:=\left[t_{0}, T\right]$, where $A, B \in C(J$, $\left.\mathbb{R}^{m \times m}\right), q \in \mathbb{R}^{m}$, is said to be of index 1 or transferable (see [4]) if there exists a smooth projection $Q \in C^{1}\left(J, \mathbb{R}^{m \times m}\right)$ onto $\operatorname{Ker} A(t)$ such that the matrix $G(t)=A(t)+B(t) Q(t)$ is nonsingular for all $t \in J$. It is proved that the index 1 property (transferability) of linear DAEs does not depend on the choice of smooth projections and is equivalent to the condition $S(t) \cap \operatorname{Ker} A(t)=\{0\}$, where $S(t):=\left\{\xi \in \mathbb{R}^{m}: B(t) \xi \in \operatorname{Im} A(t)\right\}$.

A similar result can be established for LIDEs, namely, the following lemma.

Lemma 2.1. The matrix $G_{n}:=A_{n}+B_{n} Q_{n-1, n}$ is nonsingular if and only if

$$
S_{n} \cap \operatorname{Ker} A_{n-1}=\{0\},
$$

where, as in the DAE case, $S_{n}:=\left\{\xi \in \mathbb{R}^{m}: B_{n} \xi \in \operatorname{Im} A_{n}\right\}$.

The proof of Lemma 2.1 repeats that of [3, Lemma 1] with some obvious changes, and uses the fact that condition (2.3) holds if and only if $V_{n} V_{n-1}^{-1} S_{n} \cap \operatorname{Ker} A_{n}=\{0\}$.

Since condition (2.3) does not depend on the representation of connecting operators, we get the following corollary.

Corollary 2.2. The nonsingularity of $G_{n}$ does not depend on the choice of connecting operator, that is, if $Q_{n-1, n}:=V_{n-1} \tilde{Q} V_{n}^{-1}$ and $\bar{Q}_{n-1, n}:=\bar{V}_{n-1} \tilde{Q} \bar{V}_{n}^{-1}$, then both matrices $G_{n}:=$ $A_{n}+B_{n} Q_{n-1, n}$ and $\bar{G}_{n}:=A_{n}+B_{n} \bar{Q}_{n-1, n}$ are singular or nonsingular simultaneously.

Corollary 2.2 confirms that it suffices to restrict our consideration to orthogonal projections onto $\operatorname{Ker} A_{n}$, as was done in [3]. However, in the mentioned paper, a singularvalue decomposition (SVD) of $A_{n}$ is employed for constructing an orthogonal projection $Q_{n}$ onto $\operatorname{Ker} A_{n}$ and it seems not to be convenient for a further extension of the index notion to nonlinear cases. Corollary 2.2 also allows us to introduce the following notion of index 1 LIDEs, which is quite similar to that of index 1 (transferable) linear DAEs.

Definition 2.3. The LIDEs (2.2) are said to be of index 1 if, for all $n \geqslant 0$,

(i) $\operatorname{rank} A_{n}=r$;

(ii) $G_{n}:=A_{n}+B_{n} Q_{n-1, n}$ is nonsingular. 
The main difference between linear index 1 DAEs and linear index 1 IDEs is the fact that the pencil $\{A(t), B(t)\}$ in the continuous case is always of index 1 for all $t \in J$, while for $n \geqslant 1,\left\{A_{n}, B_{n}\right\}$ is not necessarily of index 1 .

Now, we describe shortly the decomposition technique for index 1 LIDEs. Performing $P_{n} G_{n}^{-1}$ and $Q_{n} G_{n}^{-1}$ on both sides of (2.2), respectively, we get

$$
\begin{gathered}
P_{n} x_{n+1}+P_{n} G_{n}^{-1} B_{n} x_{n}=P_{n} G_{n}^{-1} q_{n}, \\
Q_{n} G_{n}^{-1} B_{n} x_{n}=Q_{n}^{-1} G_{n} q_{n} .
\end{gathered}
$$

Further, denoting $u_{n}=P_{n-1} x_{n}, v_{n}=Q_{n-1} x_{n}(n \geqslant 0)$ and observing that $P_{n} G_{n}^{-1} B_{n} Q_{n-1} x_{n}=$ $P_{n} G_{n}^{-1} B_{n} Q_{n-1, n} Q_{n, n-1} x_{n}=P_{n} Q_{n, n-1} x_{n}=P_{n} Q_{n} Q_{n, n-1} x_{n}=0$, we find $P_{n} G_{n}^{-1} B_{n} x_{n}=$ $P_{n} G_{n}^{-1} B_{n} u_{n}$. Thus, (2.4) becomes an ordinary difference equation

$$
u_{n+1}+P_{n} G_{n}^{-1} B_{n} u_{n}=P_{n} G_{n}^{-1} q_{n} .
$$

Since $Q_{n} G_{n}^{-1} B_{n} Q_{n-1} x_{n}=Q_{n} G_{n}^{-1} B_{n} Q_{n-1, n} Q_{n, n-1} x_{n}=Q_{n, n-1} x_{n}=V_{n} V_{n-1}^{-1} Q_{n-1} x_{n}=V_{n} V_{n-1}^{-1} v_{n}$, (2.5) is reduced to

$$
v_{n}=V_{n-1} V_{n}^{-1}\left(Q_{n} G_{n}^{-1} q_{n}-Q_{n} G_{n}^{-1} B_{n} u_{n}\right) .
$$

Finally,

$$
x_{n}=u_{n}+v_{n}=\left(I-Q_{n-1, n} G_{n}^{-1} B_{n}\right) u_{n}+Q_{n-1, n} G_{n}^{-1} q_{n} .
$$

Thus, if (2.2) is of index 1 , then, for given $u_{0}=P_{-1} x_{0}=P_{0} x_{0}$, we can compute $u_{n+1}$, $v_{n}$, and $x_{n}(n \geqslant 0)$ by (2.6), (2.7), and (2.8), respectively. As in the DAEs case, we only need to initialize the $P_{0}$-component of $x_{0}$. Further, putting $n=0$ in (2.8) and noting that $V_{-1}=V_{0}, u_{0}=P_{-1} x_{0}=P_{0} x_{0}$, we find that a consistent initial value $x_{0}$ must satisfy a "hidden" constraint, namely, $Q_{0}\left(I+G_{0}^{-1} B_{0} P_{0}\right) x_{0}=Q_{0} G_{0}^{-1} q_{0}$.

\section{Nonlinear implicit difference equations}

We begin this section by recalling the following version of the Hadamard theorem on homeomorphism.

Theorem 3.1 [2, page 222]. Suppose $F \in C^{1}(X, Y)$ is a local homeomorphism between two Banach spaces $X, Y$ and $\zeta(R):=\inf _{\|x\| \leqslant R}\left(\left\|\left[F^{\prime}(x)\right]^{-1}\right\|\right)^{-1}$. Then if $\int_{0}^{\infty} \zeta(R) d R=+\infty, F$ is a (global) homeomorphism of $X$ into $Y$.

In particular, if $\left\|\left[F^{\prime}(x)\right]^{-1}\right\| \leqslant \alpha\|x\|+\beta$ for all $x \in X$, where $\alpha \geqslant 0, \beta>0$, then $F$ is a homeomorphism of $X$ into $Y$. Further, suppose $F=T+H$, where $T \in C^{1}(X, Y)$, $\left\|\left[T^{\prime}(x)\right]^{-1}\right\| \leqslant \gamma$, for all $x \in X$, and $\|H(x)-H(y)\| \leqslant L\|x-y\|$, for all $x, y \in X$, then if $L \gamma<1, F$ is a homeomorphism of $X$ into $Y$.

Consider a system of nonlinear IDEs

$$
f_{n}\left(x_{n+1}, x_{n}\right)=0 \quad(n \geqslant 0),
$$

where $f_{n}: \mathbb{R}^{m} \rightarrow \mathbb{R}^{m}$ are given vector functions. 
Definition 3.2. Equation (3.1) is said to be of index 1 if

(i) the function $f_{n}$ is continuously differentiable, moreover, $\operatorname{Ker}\left(\partial f_{n} / \partial y\right)(y, x)=N_{n}$, $\operatorname{dim} N_{n}=m-r$, for all $n \geqslant 0, y, x \in \mathbb{R}^{m}$, where $1 \leqslant r \leqslant m-1$;

(ii) the matrix $G_{n}=\left(\partial f_{n} / \partial y\right)(y, x)+\left(\partial f_{n} / \partial x\right)(y, x) Q_{n-1, n}(n \geqslant 0)$ is nonsingular.

Here, we put $N_{-1}=N_{0}, V_{-1}=V_{0}, Q_{-1}=Q_{0}$, and denote by $Q_{n-1, n}$ an operator connecting two subspaces $N_{n-1}, N_{n}$.

In the remainder of this paper, for the sake of simplicity, the norm of $\mathbb{R}^{m}$ is assumed to be Euclidean.

Theorem 3.3. Let (3.1) be of index 1. Moreover, suppose that

$$
\left\|G_{n}^{-1}(y, x)\right\| \leqslant \alpha_{n}\|y\|+\beta_{n}\|x\|+\gamma_{n} \quad \forall y, x \in \mathbb{R}^{m}, \forall n \geqslant 0,
$$

where $\alpha_{n}, \beta_{n} \geqslant 0, \gamma_{n}>0$ are constants. Then the problem of finding $x_{n}$ from (3.1) and the initial condition

$$
P_{0} x_{0}=p_{0}
$$

has a unique solution.

Proof. Since

$$
f_{n}\left(x_{n+1}, x_{n}\right)-f_{n}\left(P_{n} x_{n+1}, x_{n}\right)=\int_{0}^{1} \frac{\partial f_{n}}{\partial y}\left(P_{n} x_{n+1}+t Q_{n} x_{n+1}, x_{n}\right) Q_{n} x_{n+1} d t=0,
$$

equation (3.1) becomes

$$
f_{n}\left(P_{n} x_{n+1}, P_{n-1} x_{n}+Q_{n-1} x_{n}\right)=0 \quad(n \geqslant 0) .
$$

Suppose $u_{n}=P_{n-1} x_{n}(n \geqslant 0)$ is found (for $n=0, u_{0}=P_{-1} x_{0}=P_{0} x_{0}=p_{0}$ is given). We have to find $u=P_{n} x_{n+1} \in \operatorname{Im} P_{n} \subset \mathbb{R}^{r}$ and $v=Q_{n-1} x_{n} \in \operatorname{Im} Q_{n-1} \subset \mathbb{R}^{m-r}$. Define an operator $F: \mathbb{R}^{m} \rightarrow \mathbb{R}^{m}$ by $F: z:=\left(u^{T}, v^{T}\right)^{T} \mapsto f_{n}\left(u, u_{n}+v\right)$. Let $w=\left(\Delta u^{T}, \Delta v^{T}\right)^{T}$, where $\Delta u \in \operatorname{Im} P_{n}, \Delta v \in \operatorname{Im} Q_{n-1}$, then $F^{\prime}(z) w=\left(\partial f_{n} / \partial y\right)\left(u, u_{n}+v\right) \Delta u+\left(\partial f_{n} / \partial x\right)\left(u, u_{n}+v\right) \Delta v$. Consider the linearized equation

$$
F^{\prime}(z) w=q
$$

where $q \in \mathbb{R}^{m}$ is an arbitrary fixed vector. First, observe that $G_{n} P_{n}=\left(\partial f_{n} / \partial y\right) P_{n}+\left(\partial f_{n} /\right.$ $\partial x) Q_{n-1, n} Q_{n} P_{n}=\left(\partial f_{n} / \partial y\right) P_{n}=\partial f_{n} / \partial y$, hence $G_{n}^{-1}\left(\partial f_{n} / \partial y\right)=P_{n}$ and $G_{n} Q_{n}=\left(\partial f_{n} /\right.$ $\partial x) Q_{n-1, n} Q_{n}=\left(\partial f_{n} / \partial x\right) Q_{n-1, n}$, therefore $G_{n}^{-1}\left(\partial f_{n} / \partial x\right) Q_{n-1, n}=Q_{n}$, where $G_{n}, \partial f_{n} / \partial y$, $\partial f_{n} / \partial x$ are valued at $\left(u, u_{n}+v\right)$. Further, since $P_{n} \Delta u=\Delta u, \Delta v=Q_{n-1} \Delta v=Q_{n-1, n} Q_{n, n-1} \Delta v$, then by the action of $G_{n}^{-1}$ on both sides of (3.6) and using the last observations, we get

$$
\Delta u+Q_{n, n-1} \Delta v=G_{n}^{-1}\left(u, u_{n}+v\right) q .
$$


Now, applying $P_{n}$ and $Q_{n}$ to both sides of (3.7), respectively, we find $\Delta u=P_{n} G_{n}^{-1} q$ and $Q_{n, n-1} \Delta v=Q_{n} G_{n}^{-1} q$. The last equality leads to $\Delta v=V_{n-1} V_{n}^{-1} Q_{n} G_{n}^{-1} q$. Thus, (3.6) has a unique solution $w=\left(\Delta u^{T}, \Delta v^{T}\right)^{T}$. Moreover, $\|\Delta u\| \leqslant\left\|P_{n}\right\|\left\|G_{n}^{-1}\right\|\|q\|$ and $\|\Delta v\| \leqslant$ $\left\|V_{n-1} V_{n}^{-1} Q_{n}\right\|\left\|G_{n}^{-1}\right\|\|q\|$, that is, $F^{\prime}(z)$ has a bounded inverse. A simple calculation shows that $\left\|\left[F^{\prime}(z)\right]^{-1}\right\| \leqslant \omega_{n}\|z\|+\delta_{n}$, where $\omega_{n}=\sqrt{2} \rho_{n} \max \left\{\alpha_{n}, \beta_{n}\right\}, \delta_{n}=\rho_{n}\left(\gamma_{n}+\beta_{n}\left\|u_{n}\right\|\right)$, and $\rho_{n}=\left(\left\|P_{n}\right\|^{2}+\left\|V_{n-1} V_{n}^{-1} Q_{n}\right\|^{2}\right)^{1 / 2}$. By the Hadamard theorem on homeomorphism, (3.1) has a unique solution $P_{n} x_{n+1}$ and $Q_{n-1} x_{n}$ for all $n \geqslant 0$. This completes the proof of Theorem 3.3.

In the next theorem, without loss of generality, we will use orthogonal projections onto $N_{n}$, that is, $Q_{n}=V_{n} \tilde{Q} V_{n}^{T}$ and $V_{n} V_{n}^{T}=V_{n}^{T} V_{n}=I$. In this case, $Q_{n-1, n}=V_{n-1} \tilde{Q} V_{n}^{T}$ and $\left\|Q_{n}\right\|=\left\|P_{n}\right\|=\left\|V_{n}\right\|=1$.

Theorem 3.4. Suppose $f_{n}(y, x)=g_{n}(y, x)+h_{n}(y, x)$, where

(i) $g_{n}(y, x)$ is continuously differentiable, moreover

$$
\operatorname{Ker} \frac{\partial g_{n}}{\partial y}(y, x)=N_{n}, \quad \operatorname{dim} N_{n}=m-r, \quad \forall n \geqslant 0, \forall x, y \in \mathbb{R}^{m}
$$

(ii) $G_{n}(y, x)=\left(\partial g_{n} / \partial y\right)(y, x)+\left(\partial g_{n} / \partial x\right)(y, x) Q_{n-1, n}(n \geqslant 0)$ has uniformly bounded inverses, that is, $\left\|G_{n}^{-1}(y, x)\right\| \leqslant \gamma_{n}$ for all $n \geqslant 0, y, x \in \mathbb{R}^{m}$;

(iii) $h_{n}(y, x)=h_{n}\left(P_{n} y, x\right)$ for all $n \geqslant 0, y, x \in \mathbb{R}^{m}$;

(iv) $\left\|h_{n}(y, x)-h_{n}(\bar{y}, \bar{x})\right\| \leqslant L_{n}\left(\|y-\bar{y}\|^{2}+\|x-\bar{x}\|^{2}\right)^{1 / 2}$ for all $n \geqslant 0, y, x, \bar{y}, \bar{x} \in \mathbb{R}^{m}$.

Then, if $\gamma_{n} L_{n}<1 / \sqrt{2}$ for all $n \geqslant 0$, the IVP (3.1), (3.3) has a unique solution.

Proof. Using the notations of Theorem 3.3, we define two operators $T(z)=g_{n}\left(u, u_{n}+v\right)$ and $H(z)=h_{n}\left(u, u_{n}+v\right)$, where, as before, $z:=\left(u^{T}, v^{T}\right)^{T}, u:=P_{n} x_{n+1}, v:=Q_{n-1} x_{n}$, and $u_{n}:=P_{n-1} x_{n}$. From the proof of Theorem 3.3, it follows that $\left\|\left[T^{\prime}(z)\right]^{-1}\right\| \leqslant \sqrt{2} \gamma_{n}$. On the other hand, $H(z)$ is Lipschitz continuous with a Lipschitz constant $L_{n}$ and $\sqrt{2} \gamma_{n} L_{n}<1$. Thus, the mapping $F(z)=T(z)+H(z)$ is a homeomorphism of $X$ onto $Y$, therefore the IVP (3.1), (3.3) has a unique solution.

Corollary 3.5. Suppose $f_{n}(y, x)=A_{n} y+B_{n} x+h_{n}(y, x)$, where $A_{n}, B_{n} \in \mathbb{R}^{m \times m}$, and $h_{n}$ : $\mathbb{R}^{m} \times \mathbb{R}^{m} \rightarrow \mathbb{R}^{m}$ satisfy the following conditions:

(i) $\operatorname{rank} A_{n} \equiv r$ and the matrix $G_{n}=A_{n}+B_{n} Q_{n-1, n}$ is nonsingular for all $n \geqslant 0$, where $Q_{n-1, n}$ is a connecting operator of $\operatorname{Ker} A_{n-1}$ and $\operatorname{Ker} A_{n}, A_{-1}:=A_{0}$;

(ii) $h_{n}(y, x)$ is continuously differentiable, moreover

$$
\begin{gathered}
\operatorname{Ker} A_{n} \subset \operatorname{Ker} \frac{\partial f_{n}}{\partial y}(y, x) \quad \forall n \geqslant 0, \forall y, x \in \mathbb{R}^{m}, \\
\left\|h_{n}(y, x)-h_{n}(\bar{y}, \bar{x})\right\| \leqslant L_{n}\left(\|y-\bar{y}\|^{2}+\|x-\bar{x}\|^{2}\right)^{1 / 2}, \quad \forall n \geqslant 0, \forall y, x, \bar{y}, \bar{x} \in \mathbb{R}^{m} .
\end{gathered}
$$

Then, if $L_{n}\left\|G_{n}^{-1}\right\|<1 / \sqrt{2}$, the IVP (3.1), (3.3) is uniquely solvable.

It can be shown that the explicit Euler method applied to nonlinear transferable DAEs [4] leads to nonlinear index 1 IDEs. This and other problems related to connections between DAEs and IDEs will be discussed in our forthcoming paper. 


\section{Acknowledgment}

The first author thanks Prof. Shao Xiumin and Prof. Liang Guoping for their hospitality during his visit to the Institute of Mathematics, Chinese Academy of Sciences.

\section{References}

[1] P. K. Anh and L. C. Loi, On multipoint boundary-value problems for linear implicit nonautonomous systems of difference equations, Vietnam J. Math. 29 (2001), no. 3, 281-286.

[2] M. S. Berger, Nonlinearity and Functional Analysis, Lectures on Nonlinear Problems in Mathematical Analysis. Pure and Applied Mathematics, Academic Press, New York, 1977.

[3] L. C. Loi, N. H. Du, and P. K. Anh, On linear implicit non-autonomous systems of difference equations, J. Difference Equ. Appl. 8 (2002), no. 12, 1085-1105.

[4] R. März, On linear differential-algebraic equations and linearizations, Appl. Numer. Math. 18 (1995), no. 1-3, 267-292.

Pham Ky Anh: Department of Mathematics, Mechanics, and Informatics, College of Science, Vietnam National University, 334 Nguyen Trai, Thanh Xuan, Hanoi, Vietnam

E-mail address: anhpk@vnu.edu.vn

Ha Thi Ngoc Yen: Department of Applied Mathematics, Hanoi University of Technology, 1 Dai Co Viet, 10000 Hanoi, Vietnam

E-mail address: hangocyen02@yahoo.com 